\title{
Behavioural Reactivation and Subjective Assessment of the State of Vigilance-Application to Simulated Car Driving
}

\author{
Anne Bonnefond \\ Centre d'Etudes de Physiologie Appliquée, Centre National de la Recherche Scientifique, \\ Strasbourg, France \\ Joceline Rogé
}

Laboratoire de Psychologie de la Conduite, Institut National de la Recherche sur les Transports et leur Sécurité, Arcueil, France

\begin{abstract}
Alain Muzet
Centre d'Etudes de Physiologie Appliquée, Centre National de la Recherche Scientifique, Strasbourg, France
\end{abstract}

The frequency of some behaviour (such as self-centred gestures) increases during a task that leads to the occurrence of low-vigilance episodes. These gestures can be useful in stimulating oneself. A study carried out in 20 adults has enabled us to state that motor activity (recorded with an actimeter) increases with the duration of a monotonous driving task and sleep deprivation. The analysis of the scores recorded using the Karolinska Sleepiness Scale has shown that drivers can assess the deterioration of their state of vigilance according to the actual sleep preceding the driving test. Finally, the joint analysis of the subjective and objective data revealed a co-variation of these two types of indices. We discuss the stimulatory function of the motor activity in a task leading to the occurrence of low-vigilance episodes by investigating, among other things, the use, conscious or not, of this type of activity.

actimetry sleep deprivation vigilance self-centred gestures

Karolinska Sleepiness Scale stimulatory activities

\section{INTRODUCTION}

A decrease, even transitional, in the level of vigilance affects intellectual and physical abilities and therefore also people's performance [1]. The relative lack of knowledge about their state contributes to the occurrence of errors (in terms of signals missed, mistakes made or falling asleep) that are among the most frequent causes of accidents [2]. Some individuals, unconscious of the limits of their abilities, endanger not only themselves but also others.

In the scientific literature, there is a wealth of studies aimed at demonstrating the effectiveness of diverse techniques used to improve and maintain the level of vigilance of these individuals

Correspondence and requests for offprints should be sent to Anne Bonnefond, Centre d'Etudes de Physiologie Appliquée, Centre National de la Recherche Scientifique, 21 rue Becquerel, 67087 Strasbourg CEDEX, France. E-mail: <anne.bonnefond@c-strasbourg.fr>. 
throughout the (whole) performance of their task (working, driving a car). By and large, two main types of methods emerge, each one relying on a specific principle.

The first principle proposes to increase individuals' opportunity to sleep in the form of short periods of sleep, more commonly called naps. This technique, which has numerous advantages $[3,4,5,6]$, has been shown to be beneficial in fighting drowsiness, especially at the wheel $[7,8]$.

The second principle consists of stimulating the person. Many techniques are associated with it. The first one is exposure to stimulating environmental conditions (light, smells, noise, music or environmental temperature) $[9,10,11,12$, 13]. At the wheel, opening the window or listening to the radio are certainly methods used by most drivers to fight drowsiness [14, 15]. The second stimulatory technique involves the use of natural or pharmacological substances such as caffeine, amphetamines or eugregorics $[16,17,18]$. The positive effects of caffeine on the state of alertness and performance have been notably studied in car drivers [16]. The third technique consists of carrying out a secondary task that breaks the monotony of the main task [19]. This technique is directly inspired by the actual experience of car drivers who resort to a whole series of activities to keep themselves awake [20]. Finally, the last stimulatory technique consists in varying one's activity during breaks that are inserted in the main monotonous activity. Activities can be social or physical such as the execution of simple movements $[21,22]$. Simple using one's muscles from time to time is a good way to stimulate one's body and thus to reduce drowsiness and fatigue [23]. In that spirit, car and lorry drivers with a long journey ahead are highly advised, e.g., to stop regularly for a few minutes and to take a walk around their vehicle. If the physical exercise carried out during the breaks enables the individual's stimulation, one can wonder if there is a relationship between people's motor activity during a monotonous task and their state of vigilance. Several studies of the analysis of behaviour in work situations or during a monotonous driving task tend to establish this relationship.
There exist two types of behavioural activities: those necessary to the performance of the task and those that are not directly imposed by the task. The latter are called non-specific activities [24], subsidiary activities [24] or collateral activities [25].

The number and the type of behavioural activities that appear during a monotonous and prolonged activity vary in relation to the nycthemeron (day-night alternation). Overall, the frequency of behavioural activities progressively increases throughout the whole day, while at the same time, operators' performance drops [26]. Moreover the frequency of behavioural activities can fluctuate during daytime. Thus, hours during which these behavioural activities were more numerous were identified in operators [24]. The number of activities increases also between the morning and the afternoon as does the subjective feeling of fatigue [27]. This number is overall higher at night than in daytime [28]. Even the increase in behaviour with the duration of the task is more pronounced at night than during the day.

There is a consensus in the literature about the interpretation of these behavioural variations. These activities would be indications of individuals' level of arousal [27, 29, 30, 32]. The occurrence of these activities would be a defence mechanism which enables the maintenance of performance or of a stable level of output. But they would not always be efficient. There would exist a threshold (or an optimal level of vigilance) beyond which the performance would decrease while behaviour continues to increase [26]. Even if the duration of the task has an effect on behaviour and on performance in monotonous tasks, this does not prove that the individual makes use of this behaviour to try and maintain a steady level of activation.

A recent study was carried out with subjects performing a 2-hr car drive on a simulator. An analysis of the drivers' behaviour (which was recorded with cameras) and of their state of vigilance (recorded with a physiological data logger) allowed us to confirm the existence of a relationship between the occurrence of this behaviour and the variations in the level of arousal [32]. One of the behavioural category 
groups are self-centred gestures, which can be defined as movements of hands towards the body. The subjects carried out more selfcentred gestures when they had presented more pronounced signs of low vigilance (alpha and/or theta waves) in the previous minute. Thus, these authors put forward the idea that self-centred gestures constitute behaviour which enables the driver to reactivate himself.

The self-centred gestures can be recorded by filming the driver, as was the case in the study quoted earlier. They can also be localised whilst driving by having the subject wear an actimeter on each wrist, since this device enables the recording of hand movements throughout the task. If the motor activity recorded in both wrists indeed reflects the quantity of self-centred gestures, then this activity should be more important when the proposed task combines some characteristics that lead to the occurrence of episodes of drowsiness. Hence, we put forward the hypothesis that the motor activity recorded on the wrist whilst driving increases when driving is monotonous and prolonged, more so when the task is carried out with a sleep debt.

The deterioration of the state of vigilance can be shown using objective measures such as the alpha and theta indices found on an electroencephalographic record or the performance obtained in a vigilance test. This deterioration can also be perceived by the subjects themselves as it was demonstrated in the studies carried out with the Karolinska Sleepiness Scale (KSS) [33, 34]. Furthermore, the scores obtained with the KSS significantly correlate with the performance obtained in a vigilance test involving visual modality, these two measures also being carried out over a whole night without sleep [34]. All these results show the usefulness of the KSS for subjective assessment of the state of vigilance. Like Akerstedt and Gillberg [33], we put forward the hypothesis that drivers with a sleep debt are able to assess the deterioration of their own state of vigilance. We believe that the score at this scale will be higher when subjects have driven with a sleep debt than when they have slept normally before the driving test.
As far as we know, no study has shown a relationship between the presence of selfcentred gestures and the subjective perception of the state of vigilance. If self-centred gestures are indeed used to reactivate oneself, we believe that they will be more numerous as drivers estimate they are in a very low state of vigilance. Thus, the more subjects perceive a decrease in their level of vigilance (estimated with the score obtained on the KSS) between the conditions with and without sleep deprivation, the more their motor activity (recorded with the actimeter) should increase between these two conditions.

\section{METHOD}

\subsection{Subjects}

Twenty men, whose age varied between 18 and 45 years $(M=35.5, \sigma=11.3)$, took part in this experiment. The subjects were healthy and the protocol of the study had been examined and applied according to the procedure of the law on biomedical experiments in the healthy voluntary subject.

\subsection{Device}

Seated at the wheel in the driving simulator (PAVCAS; Poste d'Analyse de la Vigilance en Conduite Automobile Simulée [vigilance analysis driving simulator]; Figures 1a and 1b), the subject was assigned the task of following another vehicle whilst keeping a constant 36-m inter-vehicle distance (i.e., a distance that corresponded to one and a half lines on the right hand-side of the road). This vehicle was the only other automobile present on the motorway and it moved at a speed of $96 \mathrm{~km} / \mathrm{hr}(\sigma=5.15)$. The driving simulator consisted of a car cabin placed on a mobile base that allowed longitudinal, vertical, and rolling and pitching movements of the cabin. It was associated with an interactive visualisation unit. The road image was placed $3.5 \mathrm{~m}$ away from the driver and covered $108^{\circ}$ of the horizontal and $27^{\circ}$ of the vertical visual field. To increase the monotonous aspect of the driving task, it was carried out in the presence of 
(a)

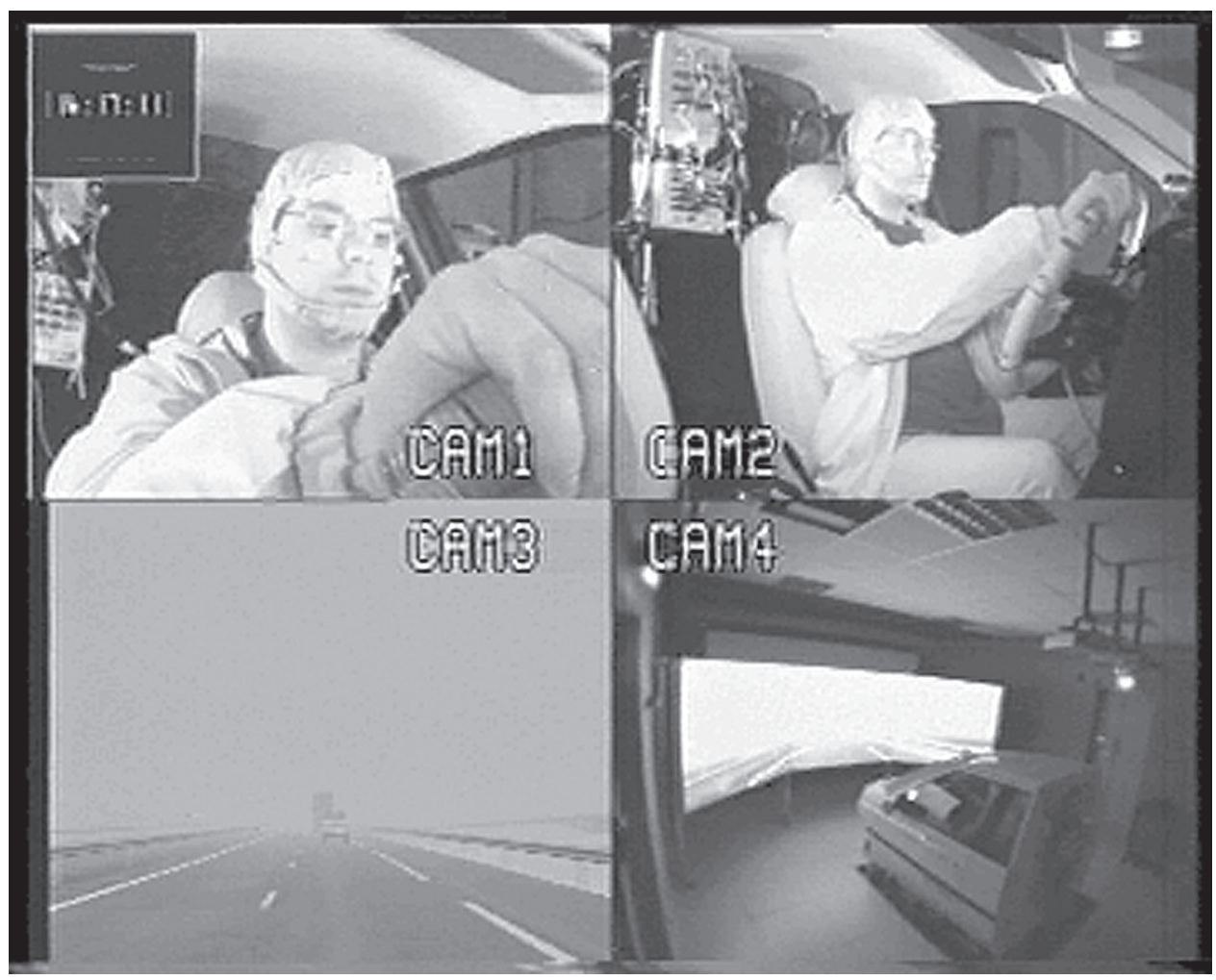

(b)

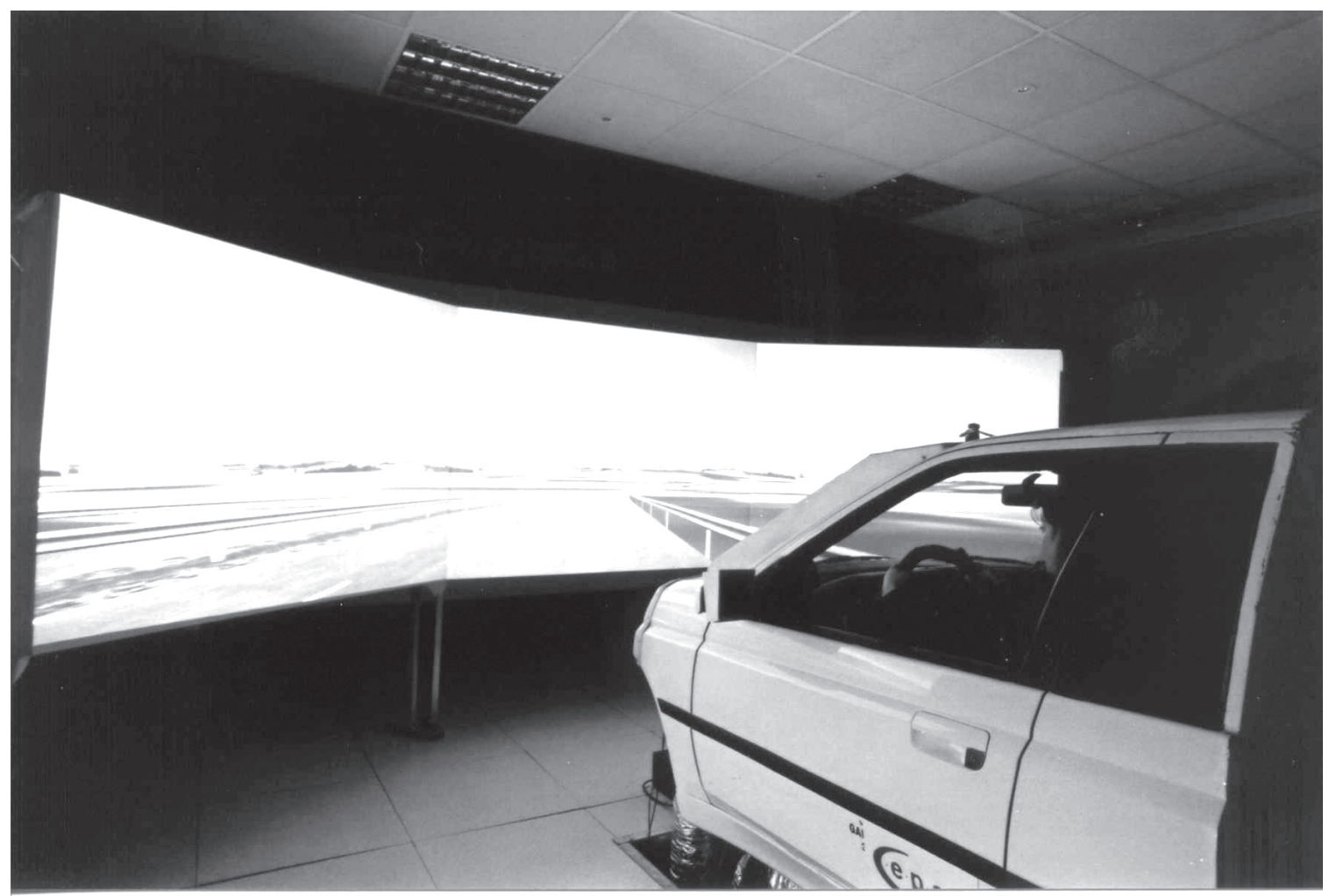

Figure 1a, Figure 1b. The experimental device: the driving simulator (PAVCAS: Poste d'Analyse de la Vigilance en Conduite Automobile Simulée [vigilance analysis driving simulator]). 
fog (located $150 \mathrm{~m}$ away). The temperature in the cabin was regulated at $21{ }^{\circ} \mathrm{C}( \pm 1)$.

During the whole car-following task, the subject wore an actimeter on each wrist (Actiwatch AW4, Cambridge Neurotechnology, version 3.24, UK). This small box, the size of a watch, is made of an accelerometer captor. The actimeter transforms any physical movements with an acceleration above a threshold of $0.1 \mathrm{~g}$ into a numeric display and records them every $15 \mathrm{~s}$. Trials before the experiment showed that those actimeters were not sensitive to the driver's movements when he manipulated the wheel during the assigned driving task. Indeed, increases in the value recorded with the actimeters did not coincide with increases in the number of movements of the wheel recorded at the same time. For all the following analyses, the first $2 \mathrm{~min}$ and the last $2 \mathrm{~min}$ of recording were excluded from the analyses because they were periods during which the driver had to manipulate the speed lever either to reach the speed of the vehicle he had to follow or to stop his vehicle at the end of the experiment. These movements were necessary for driving and did not constitute collateral activities.

\subsection{Variables}

Following the vehicle lasted for $60 \mathrm{~min}$. It was divided into two equal parts: first half-hour and second half-hour. Each subject took part in two different experimental sessions. The order of these two sessions was counterbalanced between the subjects. For the session with sleep deprivation the subject arrived at the lab at 10 p.m. and stayed awake until 8 a.m the next morning. He had the possibility to carry out quiet activities such as reading or writing in the company of an experimenter. At 8 a.m., after a light breakfast, he underwent a training session on the simulator to get familiar with both simulated driving and the assigned car-following task. At 9 a.m. he carried out the following task for $1 \mathrm{hr}$. During the session without sleep deprivation, the subject arrived at the laboratory at 8 a.m. after a complete night of sleep at home. An actimetric recording enabled us to verify the length of his nocturnal sleep. Then he carried out a training session at 8 a.m. and the monotonous driving task at 9 a.m. as in the first session with sleep deprivation. For each session, the subject filled in the KSS after the driving test [34].

The record of the actimetric data enabled us to calculate the movement index that corresponds to the percentage of 15 -s periods with an activity strictly above 0 [35]. The KSS enabled us to evaluate the driver's state of vigilance. The score on this scale can vary from 1 (very alert) to 9 (very sleepy, fighting sleep, an effort to keep awake), the intermediary level referring to a neutral state (neither awake nor sleepy).

A $2 \times 2$ analysis of variance (ANOVA) with repeated measures (the duration of driving with 2 modalities and sleep deprivation with 2 modalities) was carried out on the movement index to study the effect of sleep deprivation and driving duration. A Wilcoxon test was used to study the effect of sleep deprivation on the score obtained on the KSS. Finally, a Pearson correlation was calculated to measure the relationship between the variation of the level of vigilance between the sessions with and without sleep deprivation (the difference between the score obtained on the KSS in the sleep debt condition and the condition without a sleep debt) and the increase in actimetric data between the sessions with and without sleep deprivation (the difference between the movement index recorded in the sleep debt condition and the condition without a sleep debt).

\section{RESULTS}

\subsection{Analysis of Actimetric Data}

Sleep deprivation had a significant effect on the movement index $(F(1,19)=4.3 ; p<.05)$. The movement index was higher when the driver had not slept the night before the driving test (55.38, $\sigma=17.67)$ than when he had had a complete night's sleep (44.31, $\sigma=20.37$ ).

The duration of driving had a significant effect on the movement index $(F(1,19)=35.91$; $p<.01)$. Indeed, it increased from the first halfhour $(45.93, \sigma=18.67)$ to the second one (53.76, $\sigma=20.45)$. 


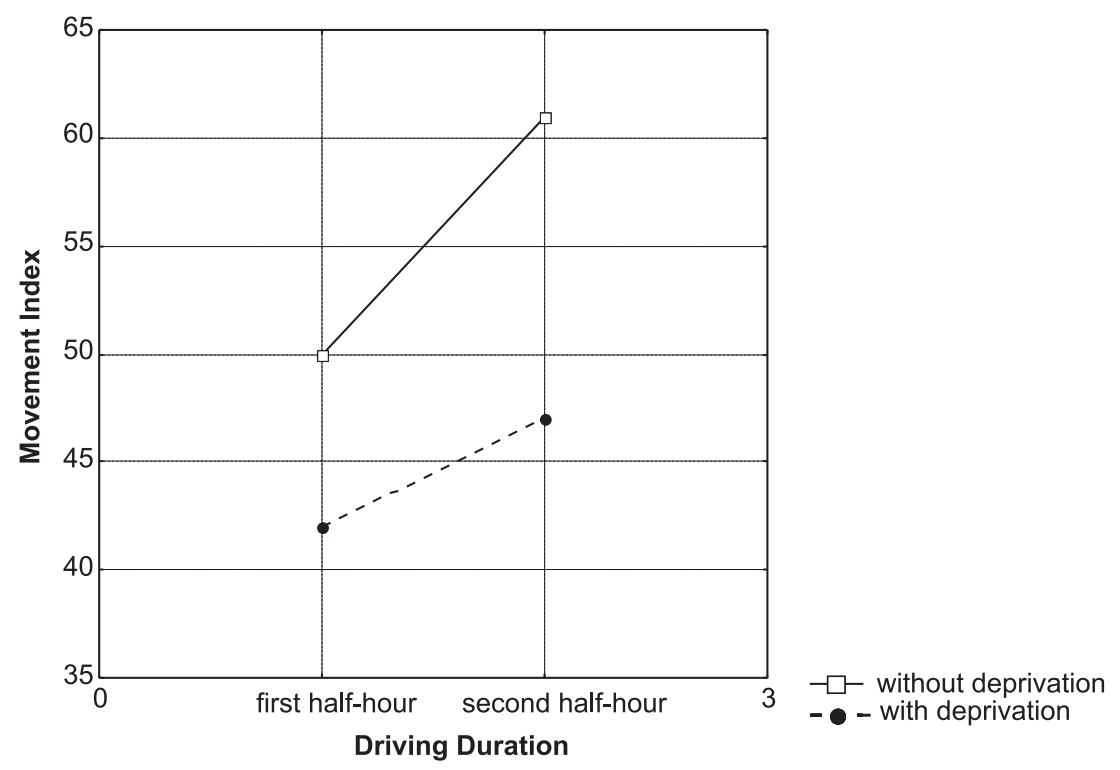

Figure 2. Index movement as a function of sleep deprivation (with sleep deprivation versus without sleep deprivation) and driving duration (first half-hour versus second half-hour).

Sleep deprivation interacted with driving duration $(F(1,19)=4.95 ; p<.05)$ on the movement index. The mean values are reported in Figure 2.

The movement index was more important when the driving task was prolonged, the more so as the subject drove with a sleep debt. The means obtained were compared with the Newman-Keuls test and the differences between these means were all significant with a probability of error of less than $5 \%$. Our first hypothesis was thus confirmed.

\subsection{Analysis of Subjective Data}

The score obtained on the KSS varied significantly as a function of sleep deprivation (Wilcoxon test $T=0 ; p<.01$ ). The driver demonstrated a more important state of drowsiness when he performed the driving test with a sleep debt (score $=8.15, \sigma=1.69$ ) than when he carried out the same task after a complete night's sleep ( score $=5.00, \sigma=2.25$ ). This result corroborated our second hypothesis.

\subsection{Joint Analysis of Actimetric and Subjective Data}

A joint analysis of the actimetric and subjective data was carried out. Each subject yielded two data points (the difference between the movement index for the sessions with and without sleep deprivation and the difference between the KSS scores for the sessions with and without sleep deprivation).

The score's variation obtained on the KSS between the two sessions correlated significantly with the variation of the movement index: $+.49, p<.05$. The increase in the score on this scale between the two sessions was associated with an increase in the activity recorded with the actimeters between the same two sessions (Figure 3). The more the subjects felt the degradation of their level of vigilance with the sleep debt (in comparison with the evaluation assessed without sleep deprivation), the more the actimetric activity increased. This result corroborated our third hypothesis.

\section{DISCUSSION}

The interaction between driving duration and sleep deprivation leads to the conclusion that motor activity increases in situations that combine several characteristics (e.g., a prolonged monotonous task, sleep deprivation) that lead to the occurrence of low-vigilance episodes. This result suggests that the driver increases his motor activity at the level of his hands, perhaps 


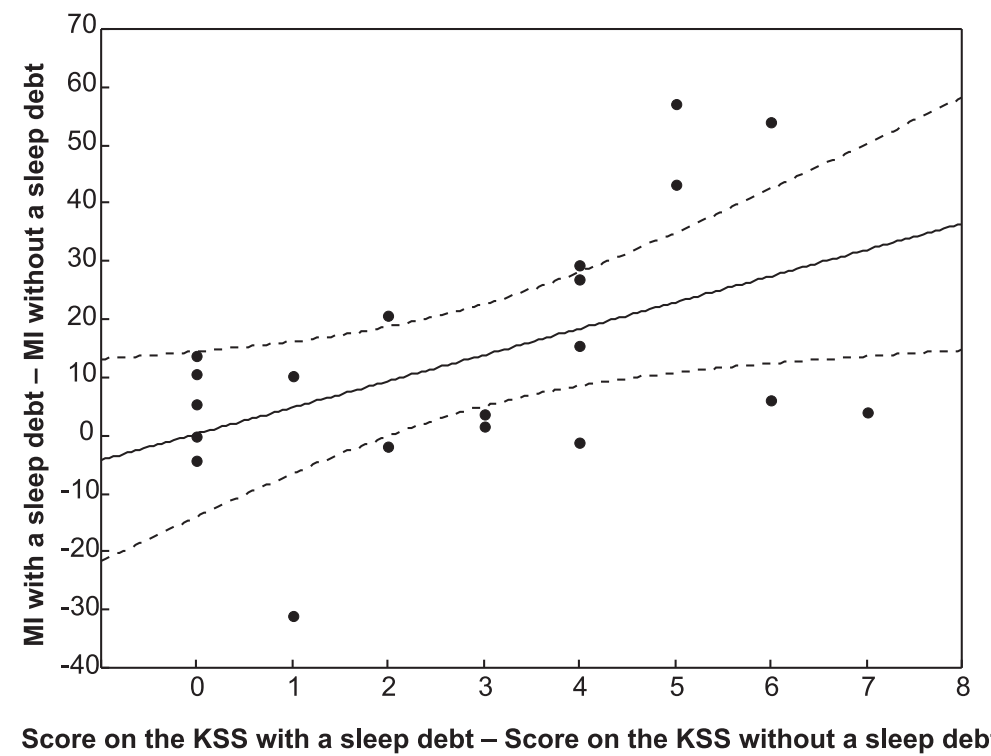

Figure 3. Variation of the movement index (MI; difference between sessions with and without sleep deprivation) as a function of the variation of the score obtained with the Karolinska Sleepiness Scale (KSS) between these 2 sessions.

in order to reactivate himself. This impact of the behavioural activity on the level of alertness has already been investigated by several researchers in the field of industry and car driving [27, 29, $30,31,32]$. It should be borne in mind that in Rogé and Muzet's [32] study the researchers noted that the increase in the driver's number of self-centred gestures systematically led to the disappearance of the signs of low vigilance on the electroencephalographic lines. The authors interpreted their results in terms of behavioural signs, precursory of low vigilance, indicative of low vigilance, or reactivating the driver. The results obtained here do not contradict that interpretation.

If the increase in the motor activity recorded by the wrist reflects the necessity for the driver to actively fight the deterioration of his state of vigilance, this could mean that the driver properly perceives the deterioration of his state. The effect of sleep deprivation on the score obtained on the KSS fully supports this explanation. The driver, even after sleep deprivation, is perfectly able to perceive and assess his state of vigilance. This finding is consistent with Akerstedt and Gillberg's [33] and Gillberg, Kecklund and Akerstedt's [34] conclusions. People can properly assess their state of vigilance over a whole night without sleeping. It should be added that they are still able to assess it in the morning following this sleep debt.

Finally, if indeed the motor activity recorded on the wrist reflects a stimulatory technique, it is logical to think that the increase in this activity is proportional to the deterioration of the state of vigilance perceived by the driver. The correlation between the index obtained on the KSS and the movement index confirms this reasoning. The more important the deterioration of the state of vigilance perceived by the driver (the difference between the self-estimation of vigilance in the sleep debt condition in comparison to the condition without sleep deprivation), the more his motor activity is important.

If the increase in motor activity is considered as a reactivation technique, the co-variation of subjective and objective data leads us to wonder about its conscious use. Indeed, one can wonder if the increase in the driver's motor activity is an automatic process (or even a reflex) or, on the contrary, if it is a result of a conscious process that can be compared with the increase in the volume of the car radio, the opening of a window [14] or the performance of ludic activities [19]. Questioning the subjects about the conscious or unconscious aspect of the variation of their 
motor activity seems essential for answering this question.

Moreover, the record and the analysis of the driving performance lead to the conclusion that this technique is effective. If increasing one's motor activity whilst driving is effective in delaying the occurrence of low-vigilance, with the prolongation of the monotonous driving task, the drivers who resort to it must also be those who present, e.g., the less important deterioration of the control of the trajectory.

It remains to be found if the effectiveness of this strategy persists with time.

Finally, drawing from these results, we believe that it would also be very interesting to compare motor activities performed by different groups of drivers in situations that lead to the occurrence of episodes of low vigilance. For example, the activity developed by healthy drivers may differ from that of drivers suffering from sleep disorders (such as people suffering from, e.g., the apnoea syndrome). Similarly, one can wonder if the motor activity of healthy drivers differs from that of drivers who have absorbed alcohol, pharmacological substances or drugs known to decrease the level of vigilance.

\section{CONCLUSION}

Beyond these teachings in terms of results, it should also be noted that this study demonstrates the advantages of recording motor activity with actimeters for this type of study. Not only is actimetry a non-cumbersome method well tolerated by the subject but, compared with video recording, it is also an economical method that enables a rapid and automated data processing.

Finally, if the technique that consists of increasing one's motor activity proves to be effective in fighting the problems of low vigilance, its use could possibly go beyond the scope of car driving and be applicable in the field of work. Indeed, in some control processes that require a high level of vigilance and sustained attention, resorting to this technique could also, in some ways, limit the decrease in workers' level of vigilance. If this activity is conscious, it would be a matter of making workers aware of this strategy in order for them to make use of it. Prior to this, a large number of questions will have to be solved, not only the conscious or automatic character of this technique, but also its effectiveness and necessary conditions.

\section{REFERENCES}

1. Folkard S, Wever RA, Wildgruber CM. Multi-oscillatory control of circadian rhythms in human performance. Nature. 1983;305:223-6.

2. Dinges DF. An overview of sleepiness and accidents. J Sleep Res. 1995;4:4-14.

3. Minors DS, Waterhouse JM. Anchor sleep as a synchronizer of rhythms on abnormal routines. Int J Chronobiol. 1981;7:165-88.

4. Matsumoto K, Morita Y. Effects of nighttime nap and age on sleep patterns of shift workers. Sleep. 1987;10:580-9.

5. Matsumoto K, Harada M. The effect of night-time naps on recovery from fatigue following night work. Ergonomics. 1994;37:899-907.

6. Saito Y, Sasaki T. The effect of length of a nocturnal nap on fatigue feelings during subsequent early morning hours. J Sci Labour. 1996;72:15-23.

7. Horne J, Reyner LA. Counteracting driver sleepiness: effects of napping, caffeine and placebo. Psychophysiology. 1996;33:306-9.

8. Reyner LA, Horne JA. Suppression of sleepiness in drivers: combination of caffeine with a short nap. Psychophysiology. 1997;34:721-5.

9. Thessing VC, Anch AM, Muehlbach MJ, Scheitzer PK, Walsh JK. Two-and-4-hour bright-light exposures differentially effect sleepiness and performance the subsequent night. Sleep. 1994;17:140-5.

10. Bonnet $\mathrm{MH}$, Arand DL. The impact of music upon sleep tendency as measured by the multiple sleep latency test and maintenance of wakefulness test. Physiol Behav. 2000;71:485-92.

11. Tassi P, Nicolas A, Dewasmes G, Eschenlauer R, Ehrhart J, Salame P, et al. Effects of noise on sleep inertia as a function of circadian placement of a one-hour nap. Percept Mot Skills. 1992;75:291-302. 
12. Dewasmes G, Signoret P, Muzet A. Un sommeil nocturne de courte durée a-t-il un effet bénéfique sur les performances cognitives du travailleur de nuit? Rapport de fin d'études. Strasbourg, France: Centre d'Etudes de Physiologie Appliquée (CNRS), 1995.

13. Foret J, Daurat A, Tirilly G. Effect of bright light at night on core temperature, subjective alertness and performance as a function of exposure time. Scand J Work Environ Health. 1998;24:115-20.

14. Maycock G. Sleepiness and driving: the experience of U.K. car drivers. Accid Anal Prev. 1997;29:453-62.

15. Reyner LA, Horne JA. Evaluation of "incar" countermeasures to sleepiness: cold air and radio. Sleep. 1998;21:46-50.

16. Horne J, Reyner L. Sleep-related vehicle accidents: some guides for road safety policies. Transportation research part F: traffic psychology and behaviour. 2001;4:63-74.

17. Shappell S, Neri DF, DeJohn C. Simulated sustained flight operations and performance, part 2: effects of dextro-methamphetamine. Mil Psychol. 1992;4:267-87.

18. Lagarde D, Batejat D. Some measures to reduce effects of prolonged sleep deprivation. Clin Neurophysiol. 1995; 25:376-85.

19. Verwey WB, Zaidel DM. Preventing drowsiness accidents by an alertness maintenance device. Accid Anal Prev. 1999;31:199-211.

20. Drory A. Effects of rest and secondary task on simulated truck-driving task performance. Hum Factors. 1985;27:201-7.

21. Lancry A, Stoklosa MH. Les effets d'une pause sur la vigilance et l'efficience au travail. Trav Hum. 1995;58:71-83.

22. Bonnet MH, Arand DL. Level of arousal and the ability to maintain wakefulness. J Sleep Res. 1999;8:247-54.

23. Horne JA, Foster SC. Can exercise overcome sleepiness? Sleep Research. 1995;24A:437.

24. Christol J, Dorel M, Quéinnec Y, de Terssac G. Horaires de travail et régulation des conduites. Trav Hum. 1979;42:211-29.
25. Delvolvé N. Les activités collatérales: repère de l'instabilité de l'homme au travail [doctoral dissertation]. Toulouse, France: University of Toulouse, Paul Sabatier; 1987.

26. Delvolvé N, Queinnec Y. Comportement d'opératrices de saisie travaillant en équipes alternantes $(2 * 8)$. Trav Hum. 1985;48:97109.

27. Knockaert S, Leconte-Lambert C, Leconte P. Impact d'une activité répétitive, monotone, continue et de longue période sur le comportement d'agents de conditionnement dans l'industrie textile. In: Proceedings of the XXXIIIth Congress of the SELF (Société d'Ergonomie de Langue Française): Temps et travail. Paris, France: SELF; 1998. p. 491-98.

28. Delvolvé N, Preteur V. Performance and organization of the activities at work as a response of human circadian variations. Behavioural Rhythms. 1986:G:177-86.

29. Welford AT. Fundamentals of skill, London, UK: Methuen; 1968.

30. Kishida K. Temporal changes of subsidiary behavior in monotonous work. J Hum Ergol. 1973;2:75-89.

31. Cosnier J. Communication non verbale et langage. Psychol Med (Paris). 1977; 9:2033-49.

32. Rogé J, Muzet A. Variations of the level of vigilance and of behavioural activities during simulated automobile driving. Accid Anal Prev. 2001;33:181-6.

33. Akerstedt T, Gillberg M. Subjective and objective sleepiness in the active individual. Int J Neurosci. 1990;52:29-37.

34. Gillberg M, Kecklund G, Akerstedt T. Relations between performance and subjective ratings of sleepiness during a night awake. Sleep. 1994;17:236-41.

35. Middelkoop H, Dam E, Doel DS, Dijk GV. 45-hour continuous quintuplesite actimetry: relations between trunk and limb movements and effects of circadian sleep-wake rhythmicity. Psychophysiology. 1997;34:199-203. 